\title{
ADVANCES IN THE SIMULATION OF AXIAL PISTON PUMPS
}

\author{
S. Mancò, N. Nervegna, *A. Lettini, L. Gilardino \\ The Fluid Power Research Laboratory \\ Dipartimento di Energetica, Politecnico di Torino \\ C.so Duca degli Abruzzi 24, 10129 Torino, Italy \\ http://www.polito.it/fluidpower \\ (E-mail: saman@polito.it, niner@polito.it, lugil@polito.it) \\ *Casappa S.p.A. \\ Via Balestrieri 1, 43044 Cavalli di Collecchio (Parma) Italy \\ http://www.casappa.com \\ (E-mail: lettinia@casappa.com)
}

\begin{abstract}
The present paper details advances attained in the modelling and simulation of axial piston pumps. The simulation environment is AMESim and new in-house developed submodels allow a quantitative appraisal of exchanged forces inside the pump. Care is devoted to the analysis and modelling of the port plate geometry that significantly contributes to the flow ripple. An advantage of the proposed simulation approach lies in the fact that pump geometry features are accounted "parametrically", this contributing flexibility while assessing and comparing performance of different pump designs. Experimental studies are also presented and critical analysis of results while serving the purpose of validating specific aspects of predictive simulations also allow to stress the limits involved in the modelling phase.
\end{abstract}

\section{KEY WORDS}

Axial piston pump, simulation, torque limiter, pump internal forces

\section{NOMENCLATURE}

$A_{p j} \quad:$ effective surface normal to the $j$ axis

$B D C$ : bottom dead centre

$D \quad$ : internal diameter of a pipe

$D_{p} \quad$ : piston diameter

$F_{0} \quad$ : spring preload

$g \quad:$ acceleration of gravity

$k \quad$ : spring stiffness

$l \quad:$ spring length under compression

$L \quad$ : length

$m \quad$ : mass

$N \quad$ : number of pistons

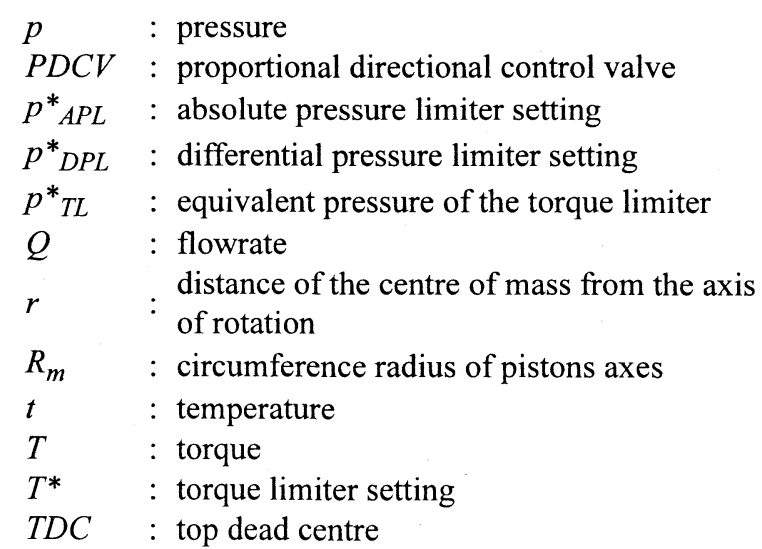




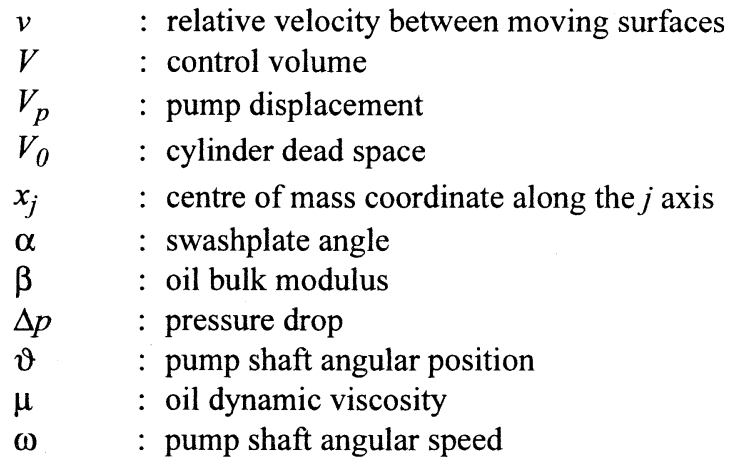

Subscripts:

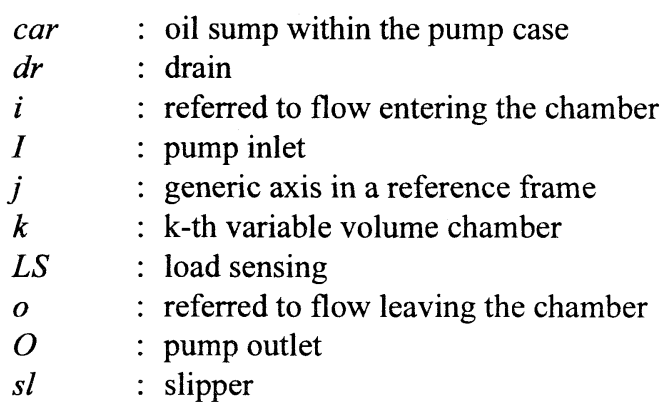

\section{INTRODUCTION}

Simulation is becoming increasingly acknowledged in the design and development phases of fluid power systems. A mathematical model descriptive of system behaviour becomes, through validation, an efficient design tool. Prototype development time can be shortened through simulation driven experiments.

Over the years numerous research studies addressed axial piston pumps. At different levels of detail the hydraulic model has drawn attention followed and complemented by analyses of internal forces. Only in recent years efforts have also been directed to tribological aspects to account for the complex interactions occurring within these units Caspar [2], Puma (DSH plus) [3]. As knowledge progresses, either oversimplified or overspecific contributions can be found at its boundaries.

The scope of this research work is to detail features and capabilities of a simulation model developed over the past four years [1]. An approximate approach is followed that neglects tribologic aspects and considers constant gap heights and uniform temperature distributions. The entire simulation is framed in the standard AMESim environment [7] (the majority of submodels has been developed and tested in house through the AMESet facility [8]).

The simulation model is specifically oriented to the Casappa LVP48 pump (max displacement $7.23 \mathrm{~cm}^{3} / \mathrm{rad}, 9$ pistons), but it has been so conceived to be easily adapted to different pump geometries. Three controls are included: absolute and differential pressure limiters [1] and a torque limiter.

\section{MATHEMATICAL MODEL}

From an hydraulic point of view the entire pump can be described by volumes within the pump (suction, delivery, case and variable chambers), expressing hydraulic capacities, and restrictors (inlet, delivery and drain ports), expressing hydraulic resistances. A vector approach is used (as in [4]).

As to volumes, the basic law governing hydraulic capacities is the continuity equation:

$$
\frac{d p}{d \vartheta}=\frac{\beta}{V \omega}\left(Q_{i}-Q_{o}-\omega \frac{d V}{d \vartheta}\right)
$$

For variable volume chambers, under the hypothesis of steady state conditions $(d \alpha / d t=0)$, we have:

$$
\left\{\begin{array}{c}
V\left(\vartheta_{k}\right)=\frac{\pi D_{p}^{2}}{4} \cdot R_{m} \tan \alpha \cdot\left(1-\cos \vartheta_{k}\right)+V_{0} \\
\frac{d V}{d \vartheta}\left(\vartheta_{k}\right)=\frac{\pi D_{p}^{2}}{4} \cdot R_{m} \tan \alpha \cdot \sin \vartheta_{k}
\end{array}\right.
$$

where $\vartheta_{k}=\vartheta-(k-1) \cdot 2 \pi / N$.

The dead space $V_{0}$ varies with the swashplate angle $\alpha$, since piston stroke is reduced with pump displacement. Flow losses are primarily due to two effects: (i) compressibility of the working fluid; (ii) flow losses through clearances. The first effect is accounted for through the fluid bulk modulus and the elasticity of containment walls in pipelines. To deal with the second effect clearances equivalent to real ones have been introduced according to the following list:

- between the port plate and the barrel leakage flows exist among delivery, inlet and case;

- in each piston - cylinder bore assembly;

- in the slipper and swashplate pair.

In equivalent terms, all clearances are considered as laminar restrictors of rectangular geometry. As to the last contribution, this is evaluated based on geometric features shown in Fig. 1, considering that the same flow goes

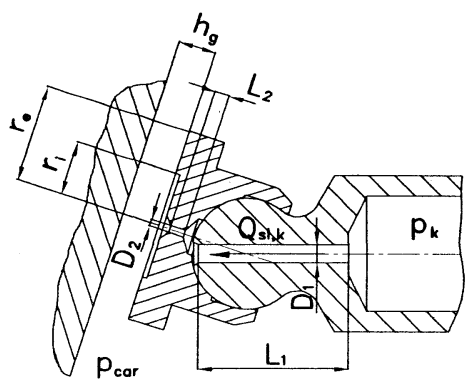

Figure 1: Hydraulic model of piston-slipper-swashplate 
through two circular section ducts within the piston and, respectively, the slipper to end up radially through the swashplate - slipper clearance:

$$
Q_{s l, k}=\frac{\pi h_{g}{ }^{3}}{6 \mu \ln \frac{r_{e}}{r_{i}}+128 \mu h_{g}{ }^{3}\left(\frac{L_{1}}{D_{1}^{4}}+\frac{L_{2}}{D_{2}^{4}}\right)} \cdot\left(p_{k}-p_{c a r}\right)
$$

Beside hydraulic quantities, the simulation model also evaluates exchanged forces within the pump. In more detail, the entire unit is broken down into its constituent components (according to the scheme shown in Fig. 2),

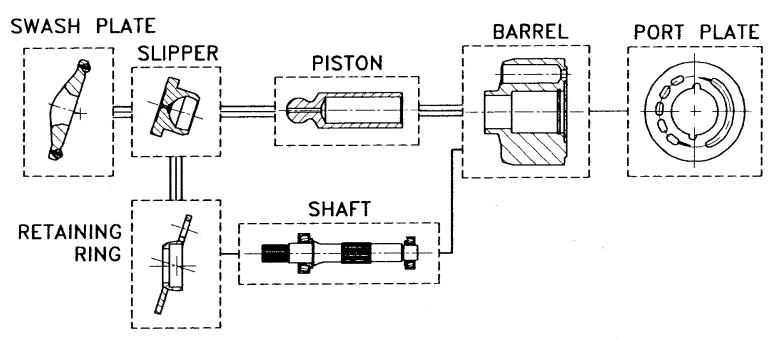

Figure 2: Mechanical model

and on each of them rotational and translational equilibrium conditions are expressed along the three axes of a cartesian reference frame. Each element of the pump has a counterpart AMESim submodel which evaluates external forces and reactions exchanged with adjacent interacting components. As to external forces the model considers the following contributions:

- fluid pressure forces: $F_{p j}=A_{p j} \cdot p$;

- weight of each component: $F_{g j}=m \cdot g$;

- inertia forces: $F_{i j}=m \cdot \ddot{x}_{j}$;

- centrifugal forces: $F_{c j}=m \cdot \omega^{2} \cdot r$;

- $\quad$ springs forces: $F_{s}=k \cdot l+F_{0}$;

- viscous friction forces: $d F=-\mu \cdot v / h \cdot d A$.

The working fluid model is based on tabular data of density and viscosity as functions of temperature and pressure. The fluid is a mineral base oil DTE25 (ISO VG32).

\section{TORQUE LIMITER}

The simulation model of the LVP48 accommodates the three limiters that can be used to control displacement according to different operating strategies. As to the torque limiter (Fig. 3), this introduces a tie between delivery pressure $p$ and the current displacement $V_{p}$, in pursuit of an ideal curve at constant torque (hyperbola $V_{p} \cdot p=T^{*}$ ). The load sensing (LS) pressure information $\left(p_{L S}\right)$, monitored at the load and downstream of the PDCV (Fig. 4) acts on the spool of the differential pressure lim-

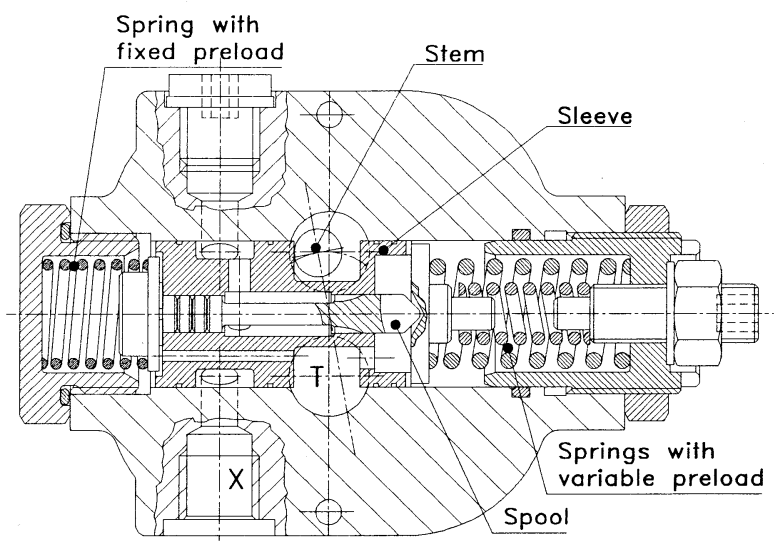

Figure 3: Torque limiter RN

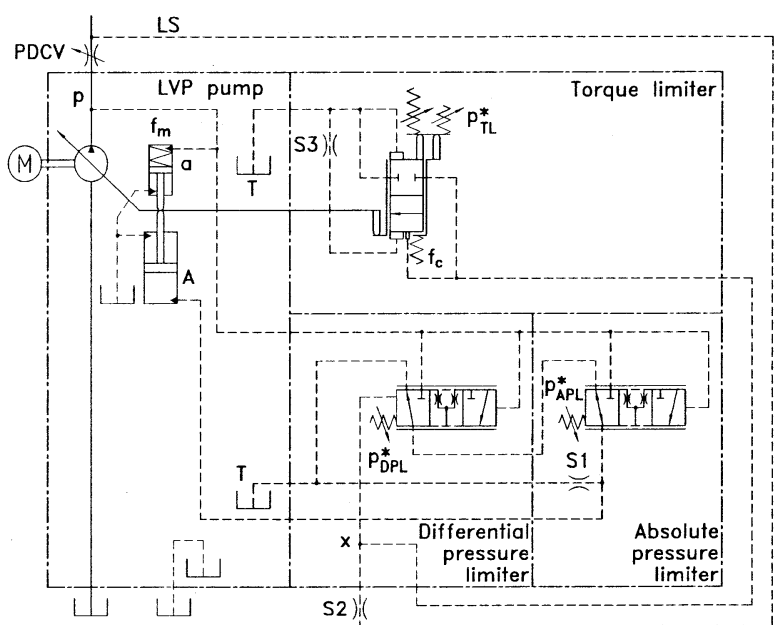

Figure 4: ISO symbol scheme of the pump-controllers system

iter (pilot $x$ ), so that delivery pressure $p$ is held above load pressure by an ideally constant amount linearly related to the differential pressure limiter setting $p^{*}{ }_{D P L}$ :

$$
p=p_{L S}+p^{*}{ }_{D P L}
$$

The same information acts on the torque limiter spool against the adjustable spring preload on the right of Fig. 3: when $p_{L S}$ is such that the limiter is active, the pilot pressure $x$ is held at $p^{*} T L$ (pressure corresponding to spring force) and, consequently, delivery pressure will be limited to:

$$
p=p^{*} T L+p^{*}{ }_{D P L}
$$

The value $p^{*} T L$ is not constant, but depends on the current displacement: in fact, the bias spring on the left of Fig. 3 steadily keeps the sleeve of the torque limiter in contact with a cylindrical stem, integral with the swashplate. As pump displacement decreases, the stem moves right and the sleeve compresses the adjustable springs with an ensu- 
ing increase of $p^{*} T L$. On the $V_{p}-p$ reference plane, the characteristic introduced by all pump controls is shown qualitatively in Fig. 5. The torque control is quasi linear and changes slope when the inner spring starts being compressed: the two linear contributions approximate the ideal hyperbola $T^{*}$.

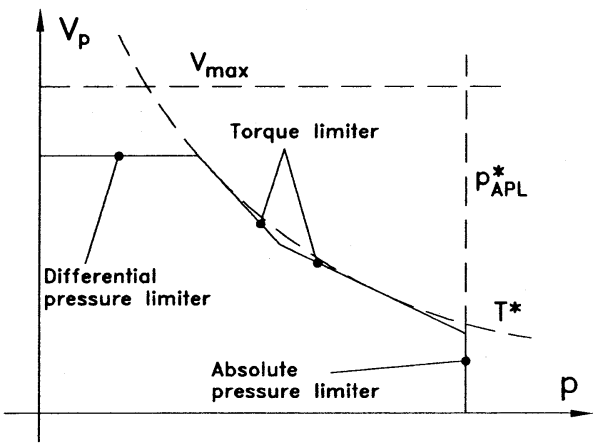

Figure 5: Displacement controls of the LVP48 pump

\section{SIMULATION MODEL AND ATTAINED RESULTS}

In Fig. 6 the AMESim diagram of the entire pump is presented. This reflects the pump structure: to the top left the hydraulic model is shown, whereas at the bottom, single mechanical items are shown (mechanic scheme of Fig. 2); to the right the three limiters (controllers) are visible.
A major advantage of the generated pump simulation model lies in its suitability as a core element (supercomponent) inside the AMESim simulation environment: it is therefore feasible to study, without unusual burdens, any circuit disposition and appraise the pump-circuit interaction.

In applications considered hereafter, the delivery pipeline downstream of the pump is modelled through distributed line models that account for wave propagation and frequency dependent friction phenomena. Circuit dynamics have in fact a noteworthy relevance on pump delivery pressure oscillations and, therefore, on generated fluid dynamic noise.

One of the more complex portions of the simulation model is relative to the determination of the flow passage areas. Beside the specific geometry interface between the port plate and the barrel of the LVP48 pump, other design solutions have been assessed that are used in axial piston units; in particular, care has been exercised in the analysis and design of relief grooves that can be widely variable in different pump models. Fig. 7 indicates parameters that precisely refer to the port plate: in this respect, dimensions and number of kidney shaped slots, relief grooves and defining angles can all be varied at will. Similarly, it is possible to reshape geometric parameters designating major pump constituents.

Therefore, pump geometry is defined parametrically, in that, prior to simulation, each dimension can be decided

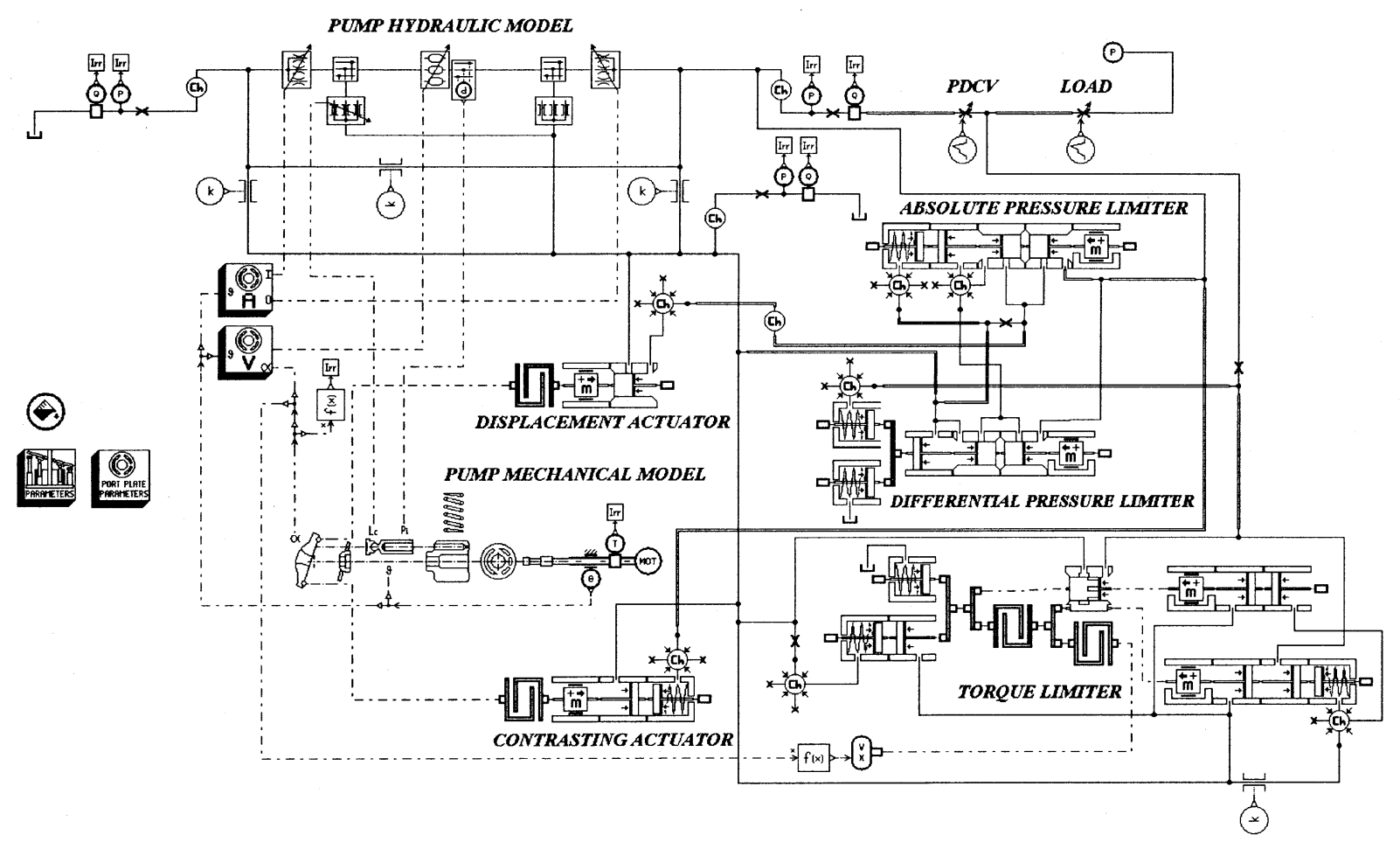

Figure 6: AMEsim circuit of the entire pump-controllers system 


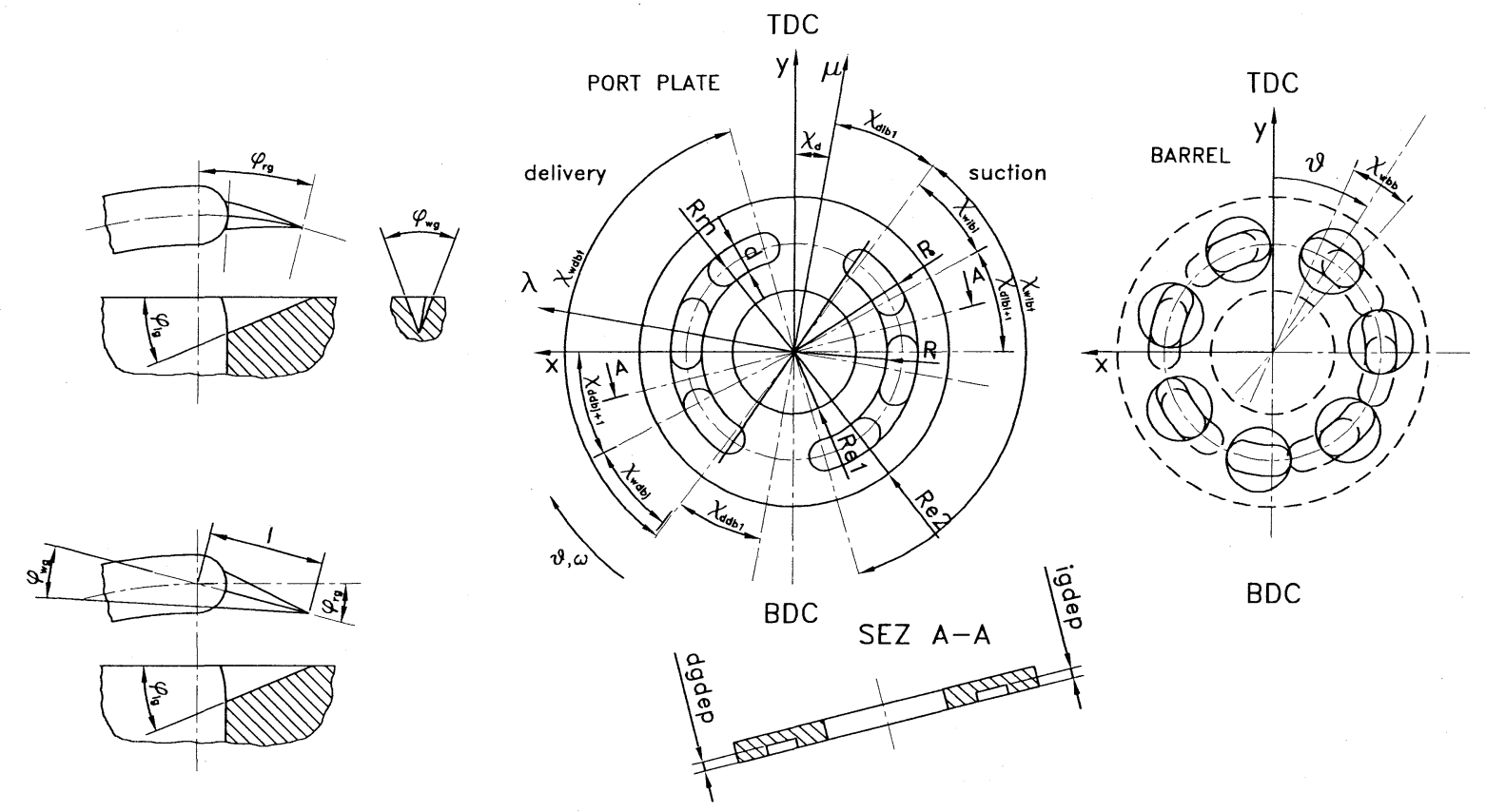

Figure 7: Geometry parameters for a generic port plate

by the user acting on a table collection of data: all ensuing quantities, derived from decided inputs, are automatically evaluated by the simulation code.

At the start of simulation, parameters are first checked for geometry consistency, then the port plate is assessed and immediately drawn on a window for visual inspection; in parallel all significant geometry features of the pump are listed for proper scrutiny (Fig. 8).

For experimental studies a rig has been assembled suitable for carrying measurements of steady state performance (Fig. 9, Fig. 15). The unit under test is boosted by a vane pump, and owing to an electrohydraulic pressure relief valve (RV1) it is possible to decide on boost pressure level. At the delivery (load) side, pressure is instead resolved by either of two means: a variable orifice $\mathrm{R}$ or an electrohydraulic pressure relief valve (RV2). The orifice location is upstream of a series of items in the delivery

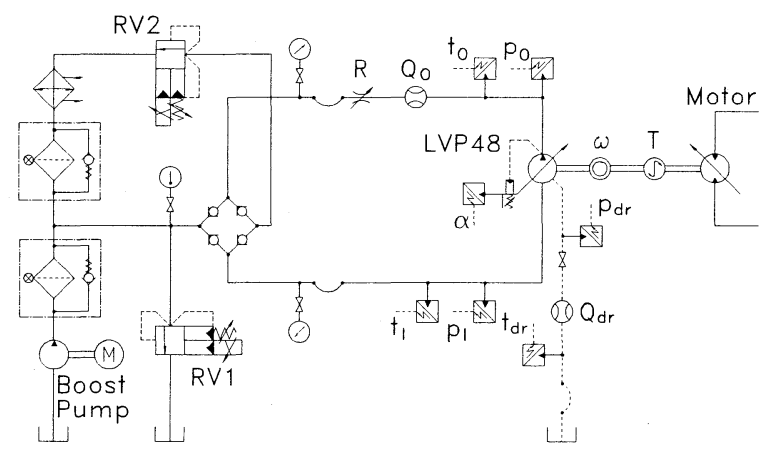

Figure 9: Test rig ISO scheme

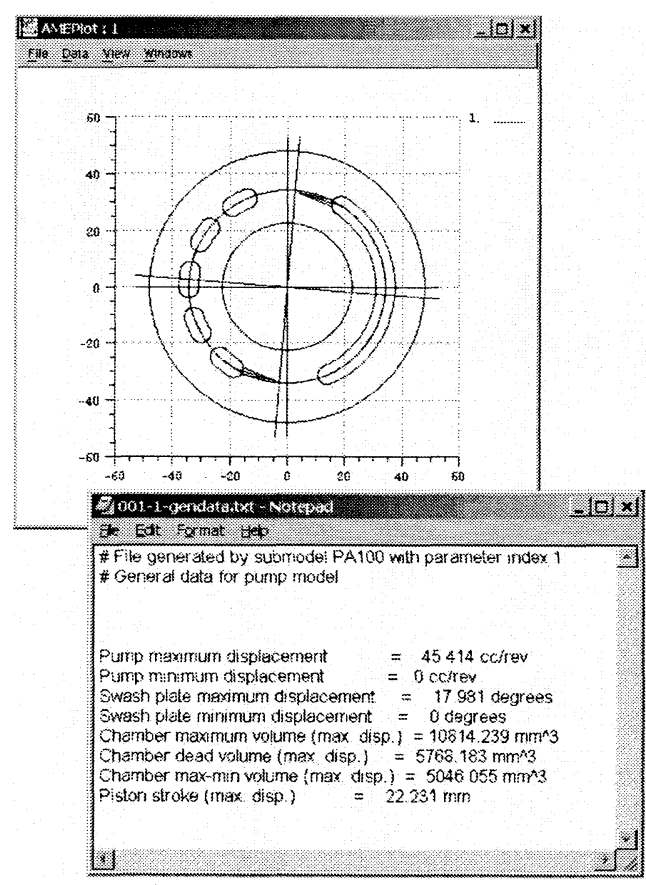

Figure 8: Dedicated windows reporting geometry features of the portplate and pump

line (a hose, check valves and ducts inside of a manifold) and it is used in tests aiming at determining the instantaneous delivery pressure: in fact, it leads to a relatively simple circuit layout formed by a constant section duct and a fixed flow restriction once the orifice flow area is pre- 
scribed. The relief valve (RV2) is instead put to use to assess the steady state pump characteristics. Discharged flow is recirculated to the suction line after passing through a heat exchanger and a filter for temperature and contamination control. Owing to check valves arranged in a Graetz bridge, it is possible to switch the high and low pressure branches according to the pump flow direction. A foremost objective of the simulation model under scrutiny is that of predicting pressure oscillations generated by the pump, so to ease and aid a design process leading to fluid dynamic noise reduction also when the unit is used in critical system applications. Hereafter, delivery pressure waveforms proper to an LVP48 are detailed as measured on the cited experimental rig and confronted with data indicated by simulation.

The delivery line consists of a pipe of length $L=0.85 \mathrm{~m}$ and inner diameter $D=19 \mathrm{~mm}$, joining the pump delivery port and the variable orifice R (Fig. 9); the working fluid temperature is controlled at $40^{\circ} \mathrm{C}$, boost pressure is 0.34 $M P a$. Tests refer to an average delivery pressure of 10 $M P a$, and different pump speeds (52.4-130.9-209.4 rad/s).
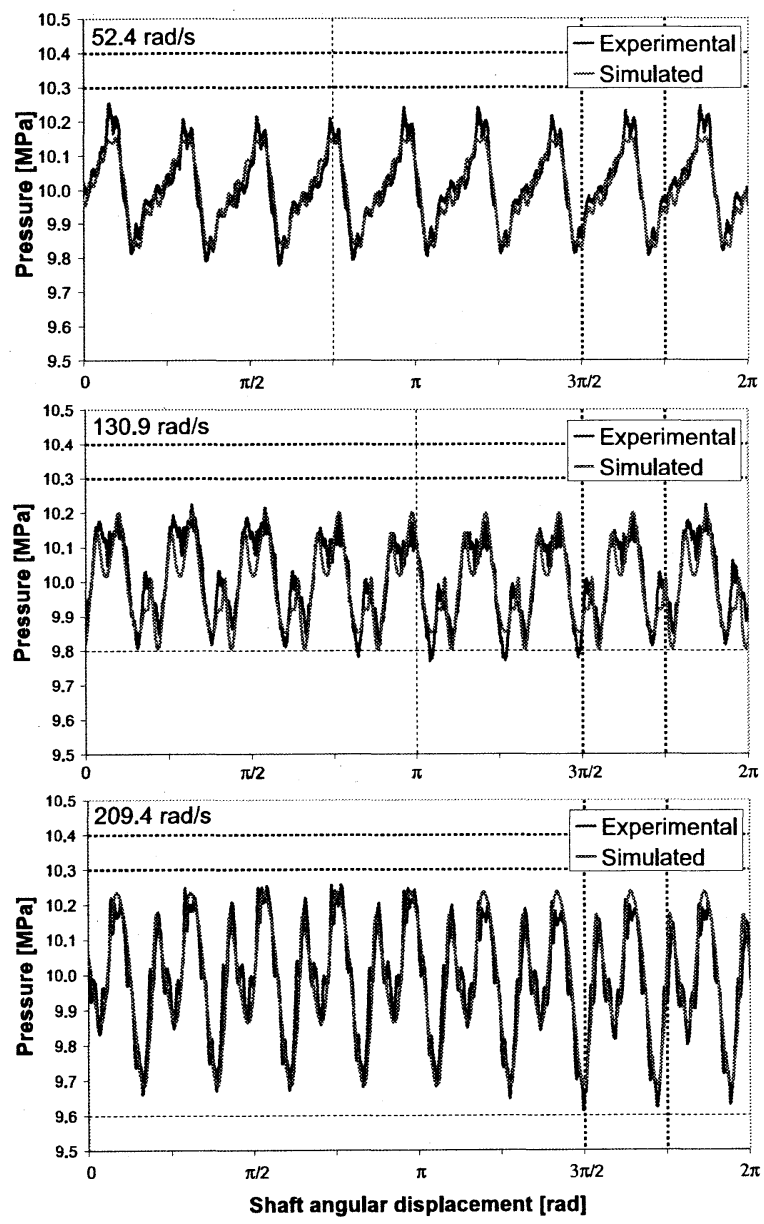

Figure 10: Delivery pressure @52.4, 130.9 and 209.4 rad/s

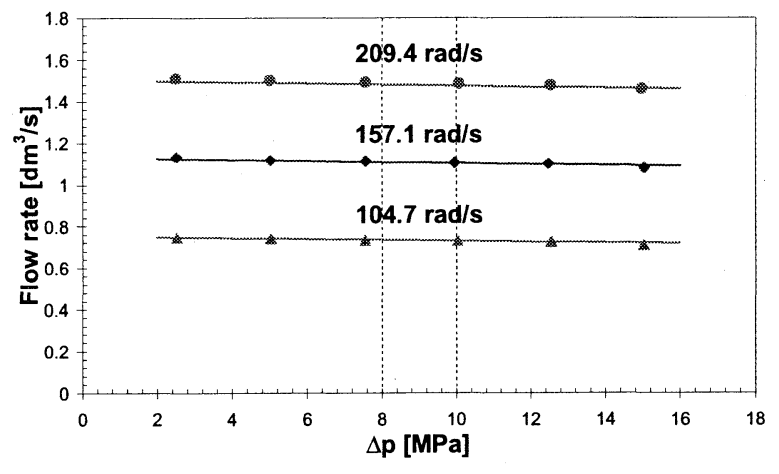

Figure 11: Steady state characteristic $Q-\Delta$ p (no controllers)

In general a good matching of experimental and simulated results can be observed in the pressure signal pattern and amplitude. The pump-circuit interaction is evidenced by a qualitatively different trend of measured pressure at different pump speeds (for more details on pump-circuit interaction and pump noise measurement see [5]). Limits of the model can be found in the prediction of high frequency harmonics (especially at top speed). Clearly visible in all three diagrams is the onset of reverse flow (backflow) occurring when each of nine chambers starts being connected with the delivery port: this event, right across the BDC (at $\pi \mathrm{rad}$ for the first chamber) originates a sudden drop of delivery pressure.

The simulation model is capable of predicting the analysed system dynamics, but may well be exercised to enquire into pump performance at steady state. Also in this case a comparison (flow characteristic in Fig. 11) is shown between simulated (continuous lines) and experimental outcomes (markers), the latter being collected on the mentioned test rig, by varying delivery pressure between 2.5 and $15 \mathrm{MPa}$ with the RV2 pressure relief valve, for different pump speeds (104.7-157.1-209.4 rad/ $s$ ); fluid temperature is held at $49^{\circ} \mathrm{C}$, and boost pressure is $0.05 \mathrm{MPa}$. Also in this case matching is good and the simulation model predicts reasonably well the dependence of pump volumetric efficiency on pressure.

Fig. 11 refers to maximum displacement and inactive controllers; Fig. 12, instead, shows the simulated pump characteristic with active controllers $(157.1 \mathrm{rad} / \mathrm{s})$. These have the following set points:

- absolute pressure limiter: $p_{A P L}^{*}=28 \mathrm{MPa}$;

- differential pressure limiter: $p^{*}{ }_{D P L}=1.4 \mathrm{MPa}$;

- torque limiter: $T^{*}=100 \mathrm{Nm}$.

The pump is set to flow saturation conditions (PDCV fully open); a progressive rise of the load leads to an increase in delivery pressure. The simulated characteristic matches the basic behaviour anticipated in Fig. 5: up to about 10 $M P a$ displacement is at maximum and delivery pressure is not sufficient in claiming the torque limiter intervention; when this happens pump displacement decreases with a 


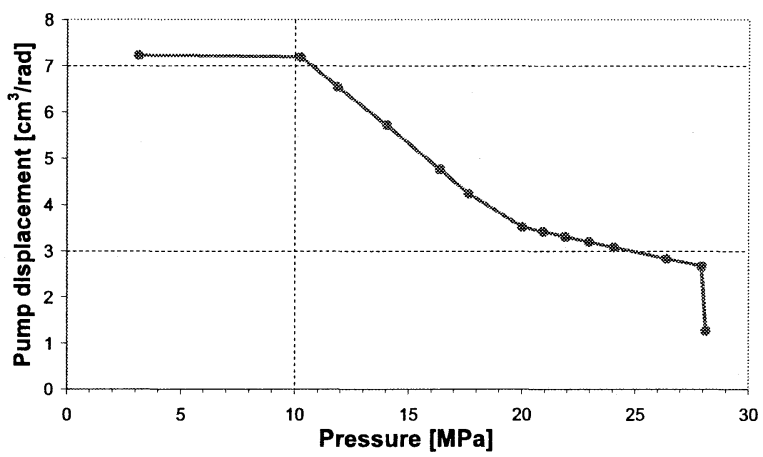

Figure 12: Simulated steady state characteristic $V_{p}-\Delta p$ (active controllers)

quasi linear trend as pressure continues to rise; at about 20 $M P a$ the torque limiter internal spring withstands compression: the characteristic stays linear but with a different slope; finally, at $28 \mathrm{MPa}$, the absolute pressure limiter takes control and impedes a further increase of pressure. An approximate analysis of forces endured by individual pump parts is quite helpful in the design process and product development; moreover, observed stresses, that bear witness of instantaneous pressure effects at distinct pump locations along with inertia effects of individual masses, may suitably serve as inputs to specific structural analysis computer codes. For instance, the port plate-barrel interface exemplifies one of the critical sites in the pump and

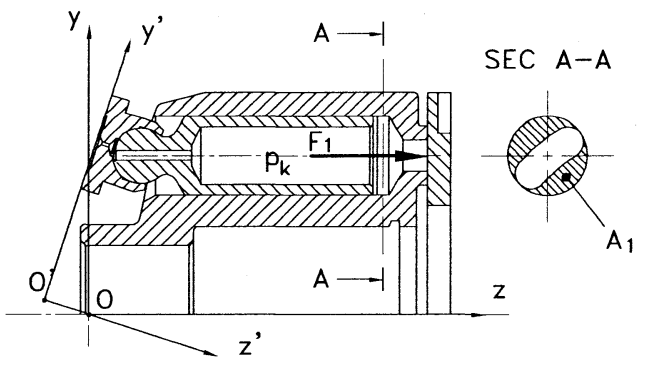

Figure 13: Pressure force pushing the barrel against the portplate

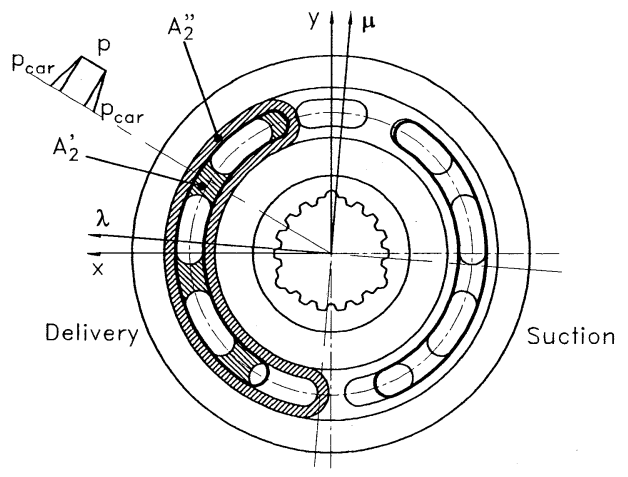

Figure 14: Pressure force separating barrel from port plate proper dimensioning of partnering surfaces is of utter importance. To this end, the simulation model evaluates counteracting pressure forces that, respectively, tend to push/separate the barrel against/from the port plate. The former $\left(F_{1}\right.$ in Fig. 13) originates from pressure $p_{k}$ acting within individual chambers (surface $A_{l}$ ), that can be considered aligned with the cylinder axis. As to the latter, consider Fig. 14, where the port plate slots are shown with a thicker trace: a first contribution originates at the delivery port where oil pressure is $p$ (surface $A_{2}^{\prime}{ }_{2}$ ), while a second occurs at the lubricated partnering surfaces of the barrel and port plate (surface $A{ }_{2}$ ); the application point of both contributions varies with the barrel angular displacement. On surfaces $A_{1}$ and $A_{2}^{\prime}$ pressure can be assumed uniform, whereas on $A{ }_{2}$ a linear trend is supposed along the radius, from value $p$ to $p_{c a r}$ according to the hypothesis of constant gap height and laminar flow regime. For simplicity, a single delivery slot deprived of relief grooves is accounted for in the model and forces at the suction port are neglected. Fig. 15 b) reports simulation results relative to the dependence on pressure of the two forces just discussed (157.1 rad/s, maximum displacement). It is important that the ratio of pushing to detaching pressure forces be greater than unity (barrel separation would otherwise occur with complete collapse of the pump) but not too high (a limit of 1.1 holds [6]), as this would lead to metal to metal contact between the barrel and the port plate. Since the model makes use of a constant gap height, the sole hydrostatic lift is accounted for, with hydrodynamic effects being ignored: in real pump performance, the two surfaces will not be parallel and the additional hydrodynamic lift will guarantee barrel equilibrium under the influence of pressure forces and of the balancing spring. Fig. $15 \mathrm{c}$ ) shows the trace of the resultant force acting on the port plate. Its position is strongly influenced by the location and dimensions of the splined coupling between barrel and shaft.

Also under remarkable stress is the swashplate, as can be inferred from Fig. 15 d,e): the resultant axial force, due to slippers action, presents, in a complete shaft revolution, a number of oscillations equal to the number of pistons. This resultant has an application point moving on a curve that mirrors that proper to the port plate with a typical butterfly shape [6]. In both instances the trace lies on the delivery side but on the swashplate it lies above the symmetry horizontal axis, and tends therefore to bring the pump to minimum displacement conditions.

Finally, Fig. 15 a) shows, at different delivery pressures, reaction forces on the shaft bearings.

\section{CONCLUSION}

In this paper principles and methods are presented that 
a)

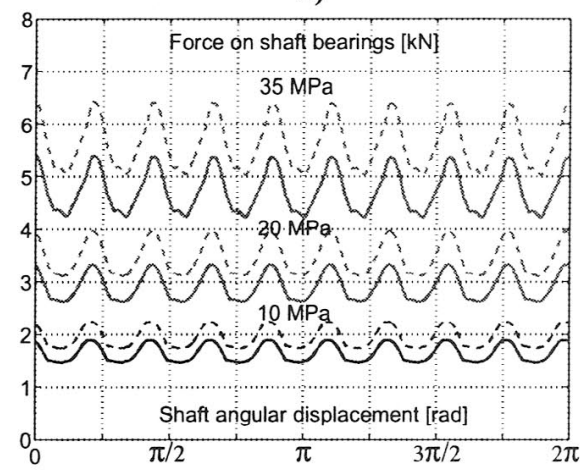

Pump mounted on the test rig

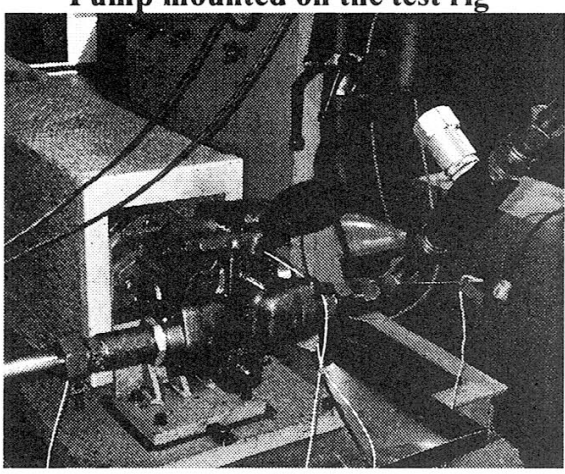

b)

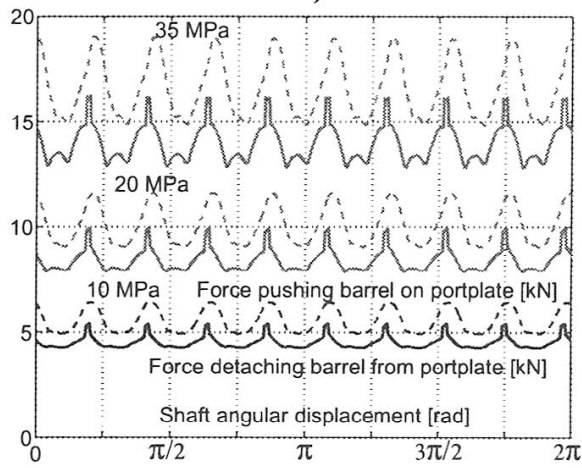

d)

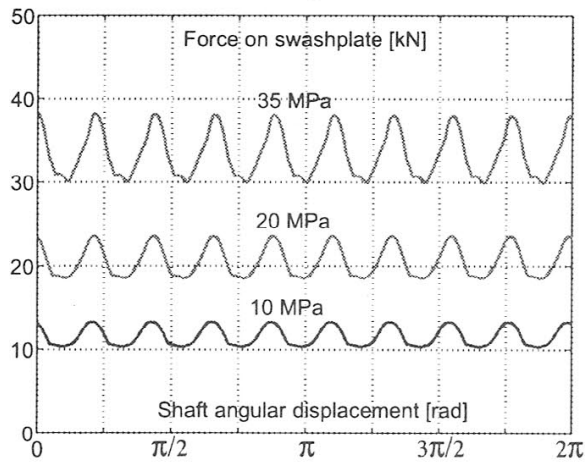

c)

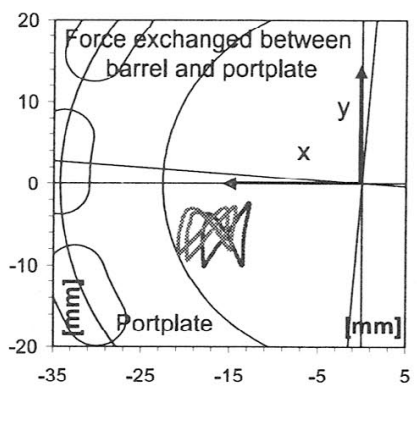

e)

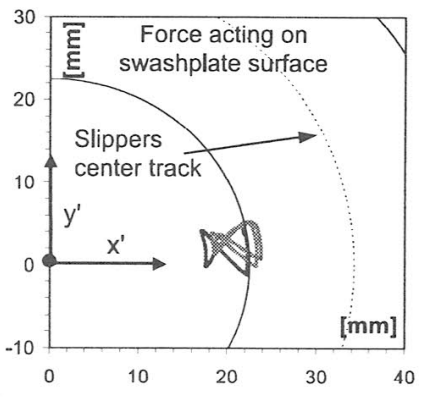

Figure 15: Simulated results of pump internal forces

form the basis of a simulation model for axial piston pumps, developed in the AMESim environment. The model, beside the pump, reflects all its displacement controllers. In particular, the torque limiter features are described. Significant results are reported relative to the Casappa LVP48 pump; some of them are compared with experimental outcomes through tests that have been performed for model validation. Simulation potential is also highlighted especially for timing analysis and pump internal forces assessment. The model that can be merged with any circuit layout in AMESim is still in development but already constitutes a dependable and powerful tool for analysis and design of axial piston pumps.

\section{ACKNOWLEDGMENTS}

The present work has been performed under a research contract with Casappa SpA. Authors acknowledge permission of publishing the present material.

\section{REFERENCES}

1. Gilardino L.; Mancò S.; Nervegna N.; Viotto F.: "An experience in simulation: the case of a variable displacement axial piston pump", The 4th JHPS International
Symposium, iss. 109, pp. 85-91, Tokyo, 1999.

2. Wieczoreck U.; Ivantysynova M.: "CASPAR - a computer-aided design tool for axial piston machines", PTMC 2000, Bath, UK, Professional Eng. Publishing, 2000.

3. Deeken M.; "Displacement unit simulation in DSHplus", Ölhydraulik und Pneumatik 45 (2001), Nr. 2

4. Mancò S.; Nervegna N.; Rundo M.: "Gerotor Lubricating Oil Pump for IC Engines", SAE International Fall Fuels \& Lubricants Meeting \& Exposition, San Francisco, SAE paper 982689 and 1998 SAE Transactions (Journal of Engines).

5. Edge K.A.; Johnston D.N.: "The 'secondary source' method for the measurement of pump pressure ripple characteristics", Proceedings of the Institution of Mechanical Engineers - Part A: Power and Process Engineering, Vol. 204, pp. 33-46, 1990.

6. Ivantysyn J.; Ivantysynova M.: "Hydrostatic pumps and motors", Akademia Books International, 2000.

7. Imagine SA: AMESim version 4.0.1, June 2002.

8. Imagine SA: AMESet version 4.0.1, June 2002. 